\title{
Bioinformatics Analysis to Identify MEG3-hsa-miR- 222-3p-NRG1/CLVS2 Axis as a Prognostic Biomarker in Prostate Cancer
}

Ke-Hao Pan

Zhongda Hospital Southeast University

Ming Chen ( $\nabla$ pankehao0319@126.com)

Zhongda Hospital Southeast University

\section{Research Article}

Keywords: prostate cancer, PTEN, ceRNA, biomarker

Posted Date: January 24th, 2022

DOI: https://doi.org/10.21203/rs.3.rs-1233865/v1

License: (c) (1) This work is licensed under a Creative Commons Attribution 4.0 International License.

Read Full License 


\section{Abstract}

Objective: To establish the competitive endogenous RNA (ceRNA) regulatory network related to phosphatase and tensin homologue (PTEN) in prostate cancer (PCa), and screen messenger RNA (mRNA), microRNA (miRNA) and long noncoding RNA (IncRNA) related to the prognosis of PCa to provide a theoretical basis for the diagnosis and prognosis of $\mathrm{PCa}$.

Methods: Transcriptome data of 498 prostate cancer samples and 52 normal prostate samples were extracted in Cancer Genome Atlas (TCGA) database and differential expression (DE) IncRNAs, miRNA and mRNA were screened. The CytoHubba plug-in was used to identify the triple regulatory network of hub genes. On this basis, the PTEN-related ceRNA regulatory network was constructed. Kaplan-Meyer method was used to explore the related factors about the prognosis of PRAD patients. The gene ontology(GO) and Kyoto Encyclopedia of Genes and Genomes(KEGG) were performed to explore functional annotation and enrichment pathways.

Results: Amount to 1918 DElncRNAs, 68 DEmiRNAs and 2920 DEmRNAs were screened. And 8 IncRNAs, 7 miRNAs and 525 mRNAs were incorporated to build the IncRNA-miRNA-mRNA triple regulatory network by Cytoscape software. The enrichment analysis of GO and KEGG showed that these interaction proteins function mainly enriched in chemotaxis, urogenital system development and cell morphogenesis involved in differentiation. Three IncRNAs (MEG3, AGAP11, and ADAMTS9-AS2), five miRNAs (miR-93-3p, miR-1413p, miR-222-3p, miR-590-3p and miR-3619-5a) and six mRNAs (NRG1, SHH, TIMP3, CLVS2, NFAT5 and ADAM22) were identified by the Cytoscape plug-in cytoHubba. Kaplan-Meier analysis screened out 2 DEmRNAs(NRG1 and CLVS2), 1 DElncRNAs(MEG3) and 1 DEmiRNA(miR-222-3p), which were associated with the prognosis of PRAD $(P<0.05)$. Finally, we established a PTEN-related MEG3-hsa-miR-222-3pNRG1/CLVS2 ceRNA network in PRAD. NRG1 and CLVS2 were related with methylation and immune infiltration in PRAD, which were high expressssion in PRAD by Immunohistochemical analysis.

Conclusion: In this study, the key RNAs related to PRAD prognosis are screened out by constructing the PTEN-related ceRNA regulatory network of PRAD, which provides a theoretical basis for the diagnosis and treatment of PRAD.

\section{Introduction}

Among all the malignant tumors, the proportion of prostate cancer( $\mathrm{PCa})$ is relatively high. In recent years, the incidence rate of $\mathrm{PCa}$ is increasing, which seriously threatens people's health and life safety ${ }^{[1]}$. Prostate cancer ranks second in male cancer mortality in the United States ${ }^{[2]}$. In early clinical diagnosis, serum prostate-specific antigen detection and digital rectal examination are mainly used to screen PCa patients, and pathological biopsy is still the gold standard of detection ${ }^{[3]}$. However, pathological biopsy is an invasive procedure, and the incidence of postoperative complications is high. In addition, it is difficult to effectively guide the treatment plan by prostate specific antigen detection and tumor staging. 
Therefore, a non-invasive biomarker with high specificity and sensitivity is urgently needed for the diagnosis and treatment of PCa patients ${ }^{[4]}$.

The pathogenic factors of prostate cancer have not been fully elucidated, heredity and genetic changes are considered to be the most important risk factors ${ }^{[5]}$. Therefore, high-throughput sequencing technology is widely used to analyze the molecular characteristics of prostate cancer, but the current understanding of its pathogenesis is still not clear. Currently, studies have found the human genome encodes lots of non coding RNAs (ncRNAs) that are not fully discovered, which can play a vital regulatory role in tumor related processes such as cell signaling ${ }^{[6]}$.

ncRNA is RNA that does not encode protein. It is mainly divided into short non coding RNA and long non coding RNA (IncRNA), which play an important role in gene transcription. LncRNA is an RNA molecule with a length of $>200$ nucleotides (NT), which not only participates in the normal biological function of the body, but also affects the genesis and progression of many tumor, like PCa. In addition to IncRNA, the discovery of microRNA (miRNA) has also played a vital role in the genesis and progression of tumor research in recent years.

It is a sort of conserved non coding single stranded small RNA with about 22 NT. When miRNA binds to messenger RNA (mRNA), it can degrade or silence its target gene by binding miRNA response element (MRE) on the target gene, so as to inhibit the translation of mRNA, which not only participates in the regulation of cell growth, aging and apoptosis, but also is closely related to all stages of life process. Currently, it is worth noting that studies have found IncRNA has the function of "Spone", which connects with miRNA and regulates the target gene of miRNA ${ }^{[7-9]}$. This is a more complex and fine molecular regulation mechanism, which is called "endogenous competitive RNA (ceRNA) Regulation Network". The core of ceRNA is that ceRNA can competitively bind the same miRNA through MRE to regulate the expression level of target genes, which shows that IncRNA can competitively bind miRNA as ceRNA to regulate $\mathrm{mRNA}^{[10]}$.

Phosphatase and tensin homology (PTEN), which in chromosome 10, is a tumor suppressor gene with dual specific phosphatase activity found after $\mathrm{p53}$. The balance of PI3K/AKT and MAPK signaling pathways is the key to the regulation of PTEN gene, which plays a vital effect in tumor inhibition through cell cycle arrest, cell apoptosis and migration. The methylation, mutation, deletion, transcriptional and post-transcriptional regulation of PTEN gene have resulted in the decrease or loss of PTEN expression, which are bound up with the genesis and progression of various malignancies ${ }^{[11]}$. PTEN gene has become the research focus on the mechanism of the genesis and progression of many malignant tumors.

In this study, differential expressed (DE) IncRNAs, miRNAs and mRNAs were obtained through PRAD transcript data in the Cancer Genome Atlas (TCGA) database and differential expression analysis of $\mathrm{PTEN}^{\text {high }}$ and PTEN ${ }^{\text {low }}$ in PRAD samples. Then, PTEN-related ceRNA regulatory network of PRAD was established, and screen out two mRNAs (NRG1 and CLVS2), one miRNA (hsa-miR-222-3p) and one 
IncRNA (MEG3) related to the prognosis of PRAD. Finally, the MEG3-hsa-miR-222-3p-NRG1/CLVS2 axis played a vital role in PRAD. Meanwhile, NRG1 and CLVS2 were related with methylation and immune infiltration in PRAD, which were high expression in PRAD by Immunohistochemical (IHC) analysis.

\section{Materials And Methods}

\section{TCGA data collection}

Based on TCGA database (https://cancergenome.nih.gov/), the transcriptome data of PRAD samples (498 cases) and normal prostate samples (52 cases) were downloaded by GDC API to obtain the miRNA and mRNA data of the samples. LncRNA and protein coding gene(PCG) were extracted by genecode V22 GTF file. The obtained data were further processed, and the low abundance mRNA, IncRNA and miRNA were removed according to the expression level of each mRNA, IncRNA and miRNA in the sample. The screening conditions: expression level $>1$. We verified the expression of PTEN in PRAD at the protein level by Human Protein Atlas (HPA) (http://www.proteinatlas.org/), and got the mutation status of PTEN from cBioPortal for Cancer Genomics (http://www.cbioportal.org/).

\section{Screening of DEmRNAs, DEmiRNAs and DElncRNAs}

The R software package DESeq2 was used for difference analysis. The screening conditions for DElncRNAs and DEmRNAs was as follows: false discovery rate $(F D R)<0.05, \| \log _{2}$ fold change $(F C) \mid>0.5$. The screening conditions for DEmiRNAs: FDR $<0.05,\left|\log _{2} \mathrm{FC}\right|>0.3$. According to this condition, 1918 DElncRNAs, 68 DEmiRNAs and 2920 DEmRNAs were obtained. The heat map and volcano map of DERNAs were drawn by $\mathrm{R}$ language.

\section{Construction of ceRNA regulatory network}

The target mRNA set for DEmiRNAs was predicted by miRTarBase (http://mirtarbase.mbc.nctu.edu.tw), MiRDB (http://mirdb.org) and TargetScan (http://www.targetscan.org/). Mircode and TargetScan database were used to predict the target IncRNA set for DEmiRNAs. The ceRNA regulatory network of IncRNA-miRNA-mRNA interaction was established on account of the above screened interacting DERNAs and the hub-ceRNA regulatory network diagram was drawn through Cytoscape 3.6.1. The sequence of DElncRNA was got through LNCipedia (https://lncipedia.org/), and the cell localization of DEIncRNA was determined on account of theirs sequence by IncLocator database (http://www.csbio.sjtu.edu.cn/bioinf/IncLocator/).

\section{Enrichment analysis of DEmRNAs}

Gene ontology (GO) and Kyoto Encyclopedia of Genes and Genomes (KEGG) analysis of DEmRNAs were performed by Metascap database to explore functional annotation and enrichment pathways, with $p<$ 0.05 representing statistically significant differences.

\section{Survival analysis of DERNAs}


Download the information of TCGA PRAD samples and map these clinical information to hub-IncRNA, hub-miRNA and hub-mRNA in ceRNA network to obtain the DERNAs expressed in clinical samples. The expression differences between the two groups were calculated and the key nodes were selected. The key nodes were analyzed by Kaplan Meier curve to screen the genes related to the prognosis of PRAD, with $p$ $<0.05$ representing statistically significant differences.

\section{Relationship between NRG1 and CLVS2 expression and methylation}

The methylation levels of NRG1 and CLVS2 were evaluated between PRAD and normal prostate tissue by UALCAN (http://ualcan.path.uab.edu/). We also explored the relationship between the expression levels of NRG1 and CLVS2 and their DNA methylation condition by MEXPRESS (http://ualcan.path.uab.edu/). The tertiary RNA sequencing (RNA-seq) of PRAD was obtained in TCGA database. The differential expression of methylation of m6A related genes in PRAD tissues with high and low NRG1 and CLVS2 gene expression was analyzed by $\mathrm{R}$ package.

\section{Relationship between NRG1 and CLVS2 and tumor immune cell infiltration}

The immune infiltrations of NRG1 and CLVS2 in PRAD was analyzed through gene module by TIMER (https://cistrome.shinyapps.io/timer/), including B cells, CD8 + T cells, CD4 + T cells, macrophages, neutrophils and dendritic cells. It was also used to evaluate the correlation between genes and the infiltration of immune cells and investigate the association between two genes copy number and immune cell infiltration levels. When $\mathrm{P}<0.05$, the difference was statistically significant.

\section{Immunohistochemistry to verify the expression of NRG1 and CVLS2 proteins in PRAD}

Two prostate cancer specimens confirmed by pathology after radical prostatectomy in Zhongda Hospital Affiliated to Southeast University in March 2021 were selected. All specimens were fixed in $10 \%$ neutral formaldehyde solution and embedded in paraffin. Envision two-step dyeing and DAB color development were used. Rabbit anti human polyclonal antibody NRG1 and Rabbit anti human polyclonal antibody CLVS2 as a primary antibody. The results showed that fine Brown particles in cytoplasm and nucleus were positive cells, and the positive staining intensity was $0,1,2$ and 3 respectively according to the staining intensity and number of positive cells. The number of positive cells $<5 \%, 5 \% \sim 35 \%, 36 \% \sim 70 \%$ and $>70 \%$ were recorded as $0,1,2$ and 3 points respectively. Take the product of the two scores for comprehensive score. The score $\leq 1$ is negative, score $2-3$ is weak positive, score 4-5 is moderate positive, and score $\geq 6$ is strong positive. In this experiment, the combination of strong and moderate positive was used as the positive criteria, and the combination of weak positive and negative was used as the negative criteria.

\section{Results}

The tumor suppressor role and prognostic value of PTEN in PRAD 
According to HPA database analysis, PTEN was over-expressed in normal prostate tissue, but downexpressed in PRAD tissue (Figures 1A and 1B). Combined with the IHC from the HPA, the same conclusion was confirmed (Figure 1G). Through survival analysis, we also found that low expression of PTEN was associated with poor overall survival (OS) in PRAD patients cohort (Figure 1C). Given the above situation, in order to further explore the potential mechanism about abnormally low expression of PTEN in PRAD, the genome and copy number of PENT were analyzed. cBioPortal analysis indicated the deletion of PTEN in the TCGA PRAD dataset (Figure 1F). Further studies showed that over one-third of PRAD samples had PTEN deletion, and their mRNA expression level was significantly lower than that exhibited diploid PTEN (Figure 1E). In addition, we also found that the copy number of PTEN was positively correlated with the mRNA expression level (Figure 1F). Therefore, we believe that the deletion of PTEN copy number in PRAD may be related to the down-expressed of its expression level.

\section{Screening results of DElncRNAs, DEmiRNAs and DEmRNAs}

Based on the above analysis, we thought that the ceRNA network associated with PTEN might be a potential prognostic model in PRAD patients. Therefore, DElncRNAs, DEmiRNAs and DEmRNAs were identified in PRAD samples with the PTEN ${ }^{\text {high }}$ and PTEN ${ }^{\text {low }}$ expression groups by TCGA database. Then, a total of 1918 DElncRNAs, 68 DEmiRNAs and 2920 DEmRNAs were screened (Figure 2A-C). Finally, the expression of 15 significant DERNAs were subjected to two-way cluster analysis, and the corresponding heat map is shown in Figure 2D-3F.

\section{Construction of ceRNA regulatory network}

The target mRNA set for DEmiRNAs was predicted by miRTarBase, MiRDB and TargetScan. Mircode and TargetScan database were used to predict the target IncRNA set for DEmiRNAs. In total, 8 IncRNAs, 7 miRNAs and 525 mRNAs in PRAD were selected to construct the ceRNA regulatory network (Figure 3A). Finally, the hub ceRNA regulatory network with three IncRNAs (MEGS, AGAP11, and ADAMTS9-AS2), five miRNAs (miR-93-3p, miR-141-3p, miR-222-3p, miR-590-3p and miR-3619-5a), and six mRNAs (NRG1, SHH, TIMP3, CLVS2, NFAT5 and ADAM22) was constructed and visualized with the help of Cytoscape plug-in cytoHubba were identified.(Figure 3B).

\section{Enrichment analysis results of DEmRNAs}

Enrichment analysis of 525 DEmRNAs was carried out by Metascape. The results of GO and KEGG showed the genes were primarily involved in chemotaxis, urogenital system development and cell morphogenesis involved in differentiation (Figure 3C).

\section{Results of analysis between the prognosis and expression of PRAD and hub-DElncRNAs, DEmiRNAs and DEmRNAs in ceRNA network}

Firstly, the expression levels of RNAs was analyzed, which from the hub ceRNA with PTEN ${ }^{\text {high }}$ and PTEN ${ }^{\text {low }}$ expression groups and in PRAD and normal prostate tissues. Three up-regulated IncRNAs 
(MEGS, AGAP11, and ADAMTS9-AS2), one up-regulated (miR-222-3p) and two down-regulated (miR-5903p, miR-141-3p) miRNAs and six up-regulated mRNAs (NRG1, SHH, TIMP3, CLVS2, NFAT5 and ADAM22) in PRAD samples was found with PTEN ${ }^{\text {high }}$ groups (Figure 4A). The three down-regulated IncRNAs (MEGS, AGAP11, and ADAMTS9-AS2), two up-regulated (miR-590-3p, miR-141-3p) and one downregulated miRNAs (miR-222-3p), and six down-regulated mRNAs (NRG1, SHH, TIMP3, CLVS2, NFAT5 and ADAM22) were found in PRAD (Figure 4B). Kaplan Meier analysis was used to further explore the correlation between DERNAs in hub-ceRNA regulatory network and the prognosis of PRAD. The results showed that one DElncRNA (MEG3), one DEmiRNAs (miR-222-3p) and two DEmRNAs (NRG1 and CLVS2) were correlated with the prognosis of PRAD (Figure 5 and Figure 6).

The IncLocator was used to analyze the subcellular localization of MEG3, AGAP11, and ADAMTS9-AS2. As shown in Figure 7A-C, only MEG3 is mainly located in the cytoplasm. These results indicated MEG3, as a ceRNA, might enhance NRG1 and CLVS2 expression by spongy miR-222-3p. In addition, expression correlation analysis indicated that there was a positive correlation between the expression of MEG3, miR220-3p, NRG1, CLVS2 and PTEN (Figure 7D-G). Therefore, the PTEN-related MEG3-miR-220-3pNRG1/CLVS2 ceRNA network was constructed.

\section{Relationship between NRG1 and CLVS2 expression and methylation}

UALCAN analysis indicated that the methylation degree of CLVS2 in normal prostate tissue was lower than that in PRAD (Figure 8C-D). In addition, methylation sites related to the expression level of NRG1/CLVS2 in their DNA sequences were found (Figure 8A-B). Normalized mRNA expression data with corresponding patients' information was collected in TCGA-PRAD. The differential expression of methylation of m6A related genes in PRAD tissues with high and low NRG1 and CLVS2 was shown in Figure 9A-B. It was suggested the expression of NRG1 and CLVS2 in prostate cancer may be regulated by methylation modification.

\section{Relationship between the expression of NRG1, CLVS2 and immune cell infiltration}

Timer database analysis showed that the expression level of NRG1 and CLVS2 in TCGA PRAD was significantly positively correlated with the infiltration level of 6 immune cells (Figure 10). These results suggest that NRG1 and CLVS2 may regulate the occurrence and development of prostate cancer by affecting immune cell infiltration.

\section{Expression of NRG1 and CLVS2 protein in prostate cancer}

As shown in Figure 11, the expression of NRG1 and CLVS2 protein was localized in the cell membrane, some nuclei and cytoplasm. The statistical results show that the comprehensive score of NRG1 and CLVS2 in prostate cancer were 4 and 5 , respectively. All reached the positive expression standard.

\section{Discussion}


Prostate cancer is one of the most common malignancies of the urinary system, which leads to a significant threat to male health. Especially, for the elderly men, its incidence is relatively high. The serum prostate-specific antigen (PSA) detection and digital rectal examination are usually adopted in the early clinical diagnosis, moreover, the histopathological biopsy is the golden criterion for the diagnosis of $\mathrm{PCa}^{[3]}$. It is difficult to determine the tumor staging by PSA testing and digital rectal examination. However, the pathological biopsy is an invasive procedure with a high incidence of postoperative complications. Therefore, our research is carried out to detect a new diagnostic marker with high specificity, sensitivity and low damage for the prostate cancer.

Heredity and genetic alterations are considered as important factors in the genesis and progression of prostate cancer. However, the potential mechanisms need to be further elucidated ${ }^{[5]}$. Previous studies have proved that ncRNA may play a vital regulatory role in the genesis and progression of tumors ${ }^{[6]}$. LncRNAs, as ncRNAs with the highest expression in PCa, can regulate gene expression through transcriptional, post-transcriptional and epigenetic mechanisms to affect the occurrence and development of prostate cancer. This suggests that IncRNAs have potential as diagnostic and therapeutic markers. The mechanism of competitive endogenous RNA (ceRNA) has been proved to be one of the important mechanisms that IncRNAs participate in the regulation of malignancies ${ }^{[13-15]}$. In addition, increasing evidence showed that PTEN gene plays an important role in tumor inhibition through apoptosis, cell cycle arrest and cell migration. In this study, we established the PTEN-related ceRNA network in prostate cancer, and further explored its prognostic value for prostate cancer.

In this research, we firstly identified the expressionn and prognosis of PTEN in prostate cancer, which showed that the low expression of PTEN was related to poor OS in PCa patients. The analysis based on the cBioPortal suggested that the deletion of PTEN copy number in PRAD might be a potential mechanism for the down-expressed of its expression level. The PTEN-related ceRNA network was established, which contained 8 IncRNAs, 7 miRNAs and 525 mRNAs. After the hub analysis, the key triple regulatory network was established including 3 IncRNAs (MEGS, AGAP11, and ADAMTS9-AS2), 5 miRNAs (miR-93-3p, miR-141-3p, miR-222-3p, miR-590-3p and miR-3619-5a), and 6 mRNA (NRG1, SHH, TIMP3, CLVS2, NFAT 5 and ADAM22). The functional enrichment analysis showed that the network significantly enriched in the "cell morphogenesis involved in differentiation". Then, the RNAs expression analysis and the survival analysis were carried out about the hub regulatory network to construct and validate the ceRNA network. Next, we conducted sub-cellular localization analysis of these three DELncRNAs, and found that MEG3 was localized in the cytoplasm, while AGAP11 and ADAMTS9-AS2 were mainly localized in the cytosol. The results indicated MEG3, as ceRNA, might enhance NRG1 and CLVS2 expression by spongy miR-222-3p. As a result, a MEG3-miR-222-3p-NRG1/CLVS2 ceRNA network which was in connection with the PCa was established.

Maternally expressed gene 3 (MEG3) is located at DLK1-MEG3 locus on human chromosome 14q32.3 ${ }^{[16]}$. The previous studies had demonstrated that MEG3, as tumor suppressor, regulated the occurrence and progression of tumors by accumulating the p53 protein ${ }^{[17]}$. In addition, MEG3 could transcripts the $1.6 \mathrm{~kb}$ 
IncRNA, which plays an important role in the process of tumor inhibition in different kinds of cancer cells, including breast, liver, gastric, lung, colorectal, cervical and ovarian tumor cells ${ }^{[18]}$. Wu et al. found that over-expression of the MEG3 could significantly inhibit the development of prostate cancer by modulating miR-9-5p/QKI-5 axis ${ }^{[19]}$. Zhou et al. proved that in the inhibition proceeding of the development of prostate cancer, MEG3 can facilitate $\mathrm{H} 3 \mathrm{~K} 27$ trimethylation of EN2 through binding to $\mathrm{EZH} 2{ }^{[20]}$. Fredsøe et al. found that the miR-222-3p could be a diagnostic and prognostic microRNA biomarker for the PCa, which was obviously downreulated in the urine samples from PCa patients as well as the PCa tissue ${ }^{[21]}$. Previous study indicated that neuregulin 1 (NRG1) rearrangements were oncogenic drivers for lots of tumors, including head and neck, renal, breast, prostate, pancreatic and endometrial cancers ${ }^{[22]}$. Especially in lung cancer, NGR1 could activate the downstream signaling including the ERK, PI3K-AKT, and NF-kB pathways by binding to ERBB3, which led to the increased tumor cell growth and proliferation ${ }^{[23]}$. And Zhang et al. proved that the anti-androgen resistance could be promoted by the tumor microenvironment-derived NRG1 in the $\mathrm{PCa}^{[24]}$. Katoh et al. performed the research which suggested that the clavesin 2 (CLV2) was a kind of neuron-specific lipid- and clathrin-binding sec 14 proteins that could regulate lysosomal morphology ${ }^{[25]}$. However, the potential influence of CLV2 on oncology has not been studied.

In our research, MEG3 and miR-222-3p were obviously more over-expressed in PCa than the normal prostate, and the higher expression of MEG3 was related to a poor outcome by survival analysis. On the contrary, the higher expression of miR-222-3p indicated a higher survival probability of PCa patients. Opposite to MEG3 and miR-222-3p, the expression of NRG1 and CLVS2 was down-regulated, and the low expression of MEG3 and high expression of NRG1 were connected with a higher survival rate in PCa patients.

Lots of studies have shown that DNA methylation has an important influence on the regulation of gene expression ${ }^{[26,27]}$. Several DNA methylation patterns had been used to explain the abnormal expression of NRG1 and CLVS2 in PRAD. Through analyzing the UALCAN, we found that NRG1 and CLVS2 had a different level of methylation in normal prostate tissues than in PRAD tissues. And the methylation sites of NRG1 and CLVS2 that were involved in their expression level were detected. These research results indicated that the poor PRAD prognosis could be in relation to the abnormal methylation.

Immune infiltration was proved to be a vital factor in the prognosis of malignant tumor ${ }^{[28-30]}$. Through the TIMER analysis, we found that the alternation of NRG1/CLVS2 gene copy numbers was associated with several immune cell infiltration levels, including B cells, CD4+ T cells, macrophages, and dendritic cells (DCs) in PRAD. There were a positive correlation between NRG1/CLVS2 expression and infiltrating levels of CD4+ T cells, macrophages, neutrophils, $B$ cells and DCs in PRAD. The results suggested these changes that were induced by the MEG3-NRG1/CLVS2 axis could affect the tumor immune microenvironment and tumor progression in PRAD, furthermore, the clinical outcomes might also be influenced by it. GO and KEGG functional enrichment analysis were conducted to explore the potential 
functions of the triple ceRNA regulatory network. And the "cell morphogenesis involved in differentiation" was especially enriched by the DEmRNAs which participated in the network.

Though our research has established the ceRNA-based triple regulatory network, which could be a potential biomarker with prognostic value in the clinical practice, there are still several drawbacks should be noticed. First of all, the final DEIncRNAs, DEmiRNAs and DEmRNAs lack further experimental studies. Secondly, the specific functions and mechanisms of the MEG3-miR-220-3p-NRG1/CLVS2 axis in PRAD need to be further explored by experiment.

In conclusion, we have built the ceRNA-based triple regulatory network (MEG3-miR-220-3p-NRG1/CLVS2) with prognostic value in the PRAD, which help us understand the association among IncRNA-miRNAmRNA better. Most importantly, our research demonstrates that the ceRNA-based triple regulatory network (MEG3-miR-220-3p-NRG1/CLVS2) can be a novel prognostic biomarker for PRAD, and it is helpful to explore the pathogenesis of PRAD and provide new thought for the clinical treatment for the prostate cancer.

\section{Conclusion}

In this study, the key RNAs related to PRAD prognosis are screened out by constructing the PTEN-related ceRNA regulatory network of PRAD, which provides a theoretical basis for the diagnosis and treatment of PRAD

\section{Abbreviations}

PCa: Prostate cancer; PTEN: pphoshatase and tensin homolog; ceRNA: competitiveendogenous RNA; ncRNAs: non coding RNAs; miRNA: microRNA; mRNA: messenger RNA; IncRNA: long none-coding RNA; TCGA: The cancer genome atlas; PRAD: Prostate adenocarcinoma; MEG3: Maternally expressed gene 3; NRG1: Neu Re Gulin 1; CLVS2: clavesin 2.

\section{Declarations}

Ethics approval and consent to participate: Our article is bioinformatics analysis. We do not need ethics approval and consent to participate

Consent for publication: All authors agree for publication.

Availability of data and material: Publicly available datasets were analyzed in this study. This data can be found here: https://portal.gdc.cancer.gov/; http://www.proteinatlas.org/ and http://www.cbioportal.org"

Competing interests: There is no conflicts of interest.

Founding: This study was funded by Medical Research Foundation of Jiangsu Province (Z2019024). 
Authors' contributions: Ke-Hao Pan is the first author. Ming Chen is the corresponding author.

Acknowledgements: None

\section{References}

1. Tao W, He T, He YD, et al. Preliminary study on the diagnostic value of urine exosomes circ_0040507 combined with PSA in the diagnosis of prostate cancer. New Medicine, 2020, 51(6): 455-458.

2. Siegel RL, Miller KD, Jemal A. Cancer statistics, 2018. CA Cancer J Clin, 2018, 68(1): 7-30.

3. Yu Q, Li P, Weng M, et al. Nano-vesicles are a potential tool to monitor therapeutic efficacy of carbon ion radiotherapy in prostate cancer. Journal of Biomedical Nanotechnology, 2018, 14(1): 168-178.

4. Huang MD, Xiao CJ, Zheng HQ. The biological characteristics of exosomes and the application of microRNA in the diagnosis and treatment of cerebral infarction. Chinese Journal of Physical Medicine and Rehabilitation, 2020 ,42(8): 764-768.

5. Attard G, Parker C, Eeles RA, et al. Prostate cancer. The Lancet, 2016, 387(10013): 70-82.

6. Zeuschner P, Linxweiler J, Junker K. Non-coding RNAs as biomarkers in liquid biopsies with a special emphasis on extracellular vesicles in urological malignancies. Expert Rev Mol Diagn, 2020, 20(2): 151-167.

7. Chen W, Yu Z, Huang W, et al. LncRNA LINC00665 promotes prostate cancer progression via miR1224-5p/SND1 axis. Onco Targets Ther, 2020, 13: 2527-2535.

8. Xiu D, Liu L, Cheng M, et al. Knockdown of IncRNA TUG1 enhances radiosensitivity of prostate cancer via the TUG1/miR-139-5p/SMC1A axis. Onco Targets Ther, 2020, 13: 2319-2331.

9. Hua JT, Ahmed M, Guo H, et al. Risk SNP-mediated promoterenhancer switching drives prostate cancer through IncRNA PCAT19. Cell, 2018, 174(3): 564-575.

10. Ye G, Guo L, Xing Y, et al. Identification of prognostic biomarkers of prostate cancer with long noncoding RNA-mediated competitive endogenous RNA network. Exp Ther Med, 2019, 17(4): 3045-3050.

11. Hollander MC, Blumenthal GM, Dennis PA. PTEN loss in the continuum of common cancers, rare syndromes and mouse models. Nat Rev Cancer, 2011, 11(6): 289-301.

12. Ping YY, Zhou Y, Hu J, et al. Dissecting the Functional Mechanisms of Somatic Copy-Number Alterations Based on Dysregulated ceRNA Networks across Cancers. Mol Ther Nucleic Acids, 2020. 21: 464-479.

13. Han Q, Li JH, Xiong JP, et al. Long noncoding RNA LINC00514 accelerates pancreatic cancer progression by acting as a ceRNA of miR-28-5p to upregulate Rap1b expression. J Exp Clin Cancer Res, 2020. 39(1): 151.

14. Yang XZ, Cheng TT, He QJ, et al. LINC01133 as ceRNA inhibits gastric cancer progression by sponging miR-106a-3p to regulate APC expression and the Wnt/ $\beta$-catenin pathway. Mol Cancer, 2018. 17(1): 126. 
15. Yu YL, Gao FB, He Q, et al. IncRNA UCA1 Functions as a ceRNA to Promote Prostate Cancer Progression via Sponging miR143. Mol Ther Nucleic Acids, 2020. 19: 751-758.

16. Wylie AA, Murphy SK, Orton TC, et al. Novel imprinted DLK1/GTL2 domain on human chromosome 14 contains motifs that mimic those implicated in IGF2/H19 regulation. Genome Res, 2000. 10(11): 1711-8.

17. Zhou YL, Zhong Y, Wang YY, et al. Activation of p53 by MEG3 non-coding RNA. J Biol Chem, 2007. 282(34): 24731-42.

18. Al-Rugeebah A, Alanazi M, Parine NR. MEG3: an Oncogenic Long Non-coding RNA in Different Cancers. Pathol Oncol Res, 2019. 25(3): 859-874.

19. Wu M, Huang YW, Chen TC, et al. LncRNA MEG3 inhibits the progression of prostate cancer by modulating miR-9-5p/QKI-5 axis. J Cell Mol Med, 2019. 23(1): 29-38.

20. Zhou YJ, Yang HQ, Xia W, et al. LncRNA MEG3 inhibits the progression of prostate cancer by facilitating H3K27 trimethylation of EN2 through binding to EZH2. J Biochem, 2020. 167(3): $295-301$.

21. Fredsoe J, Rasmussen AKI, Thomsen AR, et al. Diagnostic and Prognostic MicroRNA Biomarkers for Prostate Cancer in Cell-free Urine. Eur Urol Focus, 2018. 4(6): 825-833.

22. Drilon A, Somwar R, Mangatt BP, et al. Response to ERBB3-Directed Targeted Therapy in NRG1Rearranged Cancers. Cancer Discov, 2018. 8(6): 686-695.

23. Fernandez-Cuesta L, Thomas RK. Molecular Pathways: Targeting NRG1 Fusions in Lung Cancer. Clin Cancer Res, 2015. 21(9): 1989-94.

24. Zhang Z, Karthaus WR, Lee YS, et al. Tumor Microenvironment-Derived NRG1 Promotes Antiandrogen Resistance in Prostate Cancer. Cancer Cell, 2020. 38(2): 279-296 e9.

25. Katoh YK, Ritter B, Gaffry T, et al. The clavesin family, neuron-specific lipid- and clathrin-binding Sec14 proteins regulating lysosomal morphology. J Biol Chem, 2009. 284(40): 27646-54.

26. Chen Y, Ren B, Yang JS, et al. The role of histone methylation in the development of digestive cancers: a potential direction for cancer management. Signal Transduct Target Ther, 2020. 5(1): 143.

27. Shu WJ, Du HN, et al. The methyltransferase SETD3-mediated histidine methylation: Biological functions and potential implications in cancers. Biochim Biophys Acta Rev Cancer, 2021. 1875(1): 188465.

28. Jin Y, Chen DL, Wang F, et al. The predicting role of circulating tumor DNA landscape in gastric cancer patients treated with immune checkpoint inhibitors. Mol Cancer, 2020. 19(1): 154.

29. Yang CY, Fan $\mathrm{MH}$, Miao $\mathrm{CH}$, et al. Engineering Chimeric Antigen Receptor $\mathrm{T}$ Cells against Immune Checkpoint Inhibitors PD-1/PD-L1 for Treating Pancreatic Cancer. Mol Ther Oncolytics, 2020. 17: 571-585.

30. Zhang LM, Wang W, Wang RK, et al. Reshaping the Immune Microenvironment by Oncolytic Herpes Simplex Virus in Murine Pancreatic Ductal Adenocarcinoma. Mol Ther, 2021. 29(2): 744-761.

\section{Figures}


A

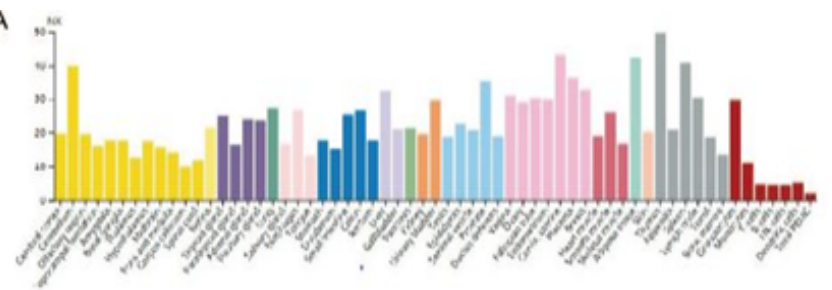

B
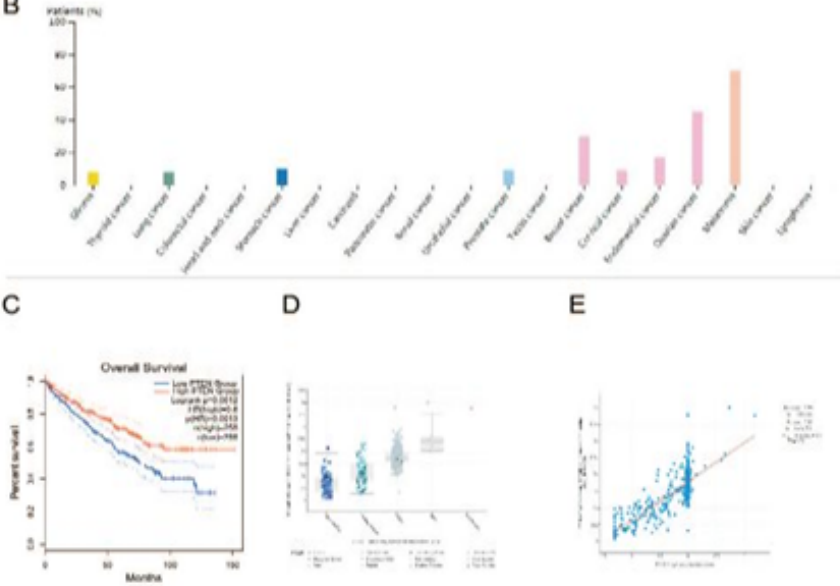

F
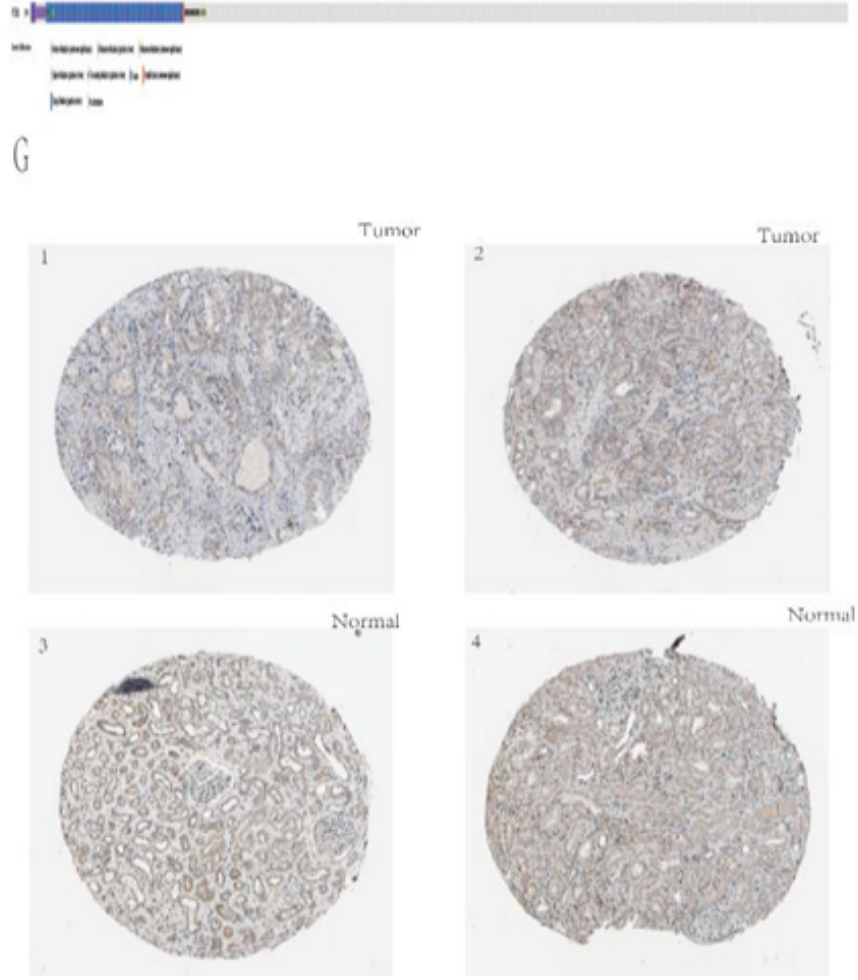

\section{Figure 1}

The tumor suppressor role of PTEN in prostate adenocarcinoma(PRAD)

(A) Expression distribution of PTEN in different normal tissues. (B) Expression distribution of PTEN in pan-cancer tissue. (C) The low and high expression of PTEN were compared using a Kaplan-Meier survival curve. (D and E) The association between PTEN copy number and mRNA expression are shown 
in the dot plot (D) and correlation plot (E) by cBioPortal. (F) The distribution of PTEN genomic alterations in TCGA PRAD is shown on a cBioPortal OncoPrint plot. $(\mathrm{G})$ Validation of the expression of PTEN on the translational level by the Human Protein Atlas database (immunohistochemistry).
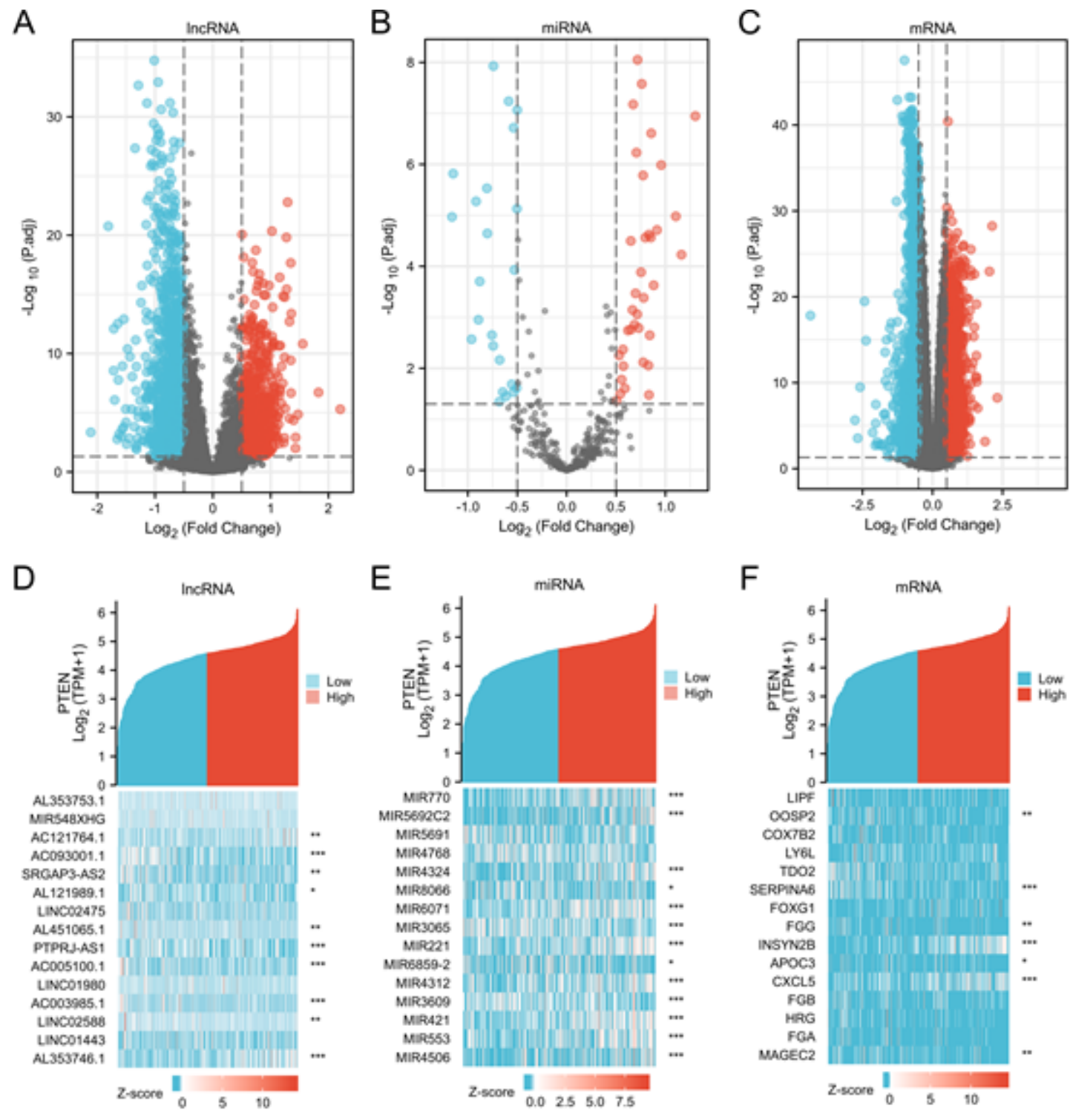

\section{Figure 2}

Volcano plots and heatmap plots of DERNAs between the expression of PTEN ${ }^{\text {high }}$ and PTEN ${ }^{\text {low }}$ in PRAD samples.

Red represents upregulated genes and blue indicates downregulated genes. The volcano plots describe (A) 1918 DElncRNAs , (B) 68 DEmiRNAs and (C) 2920 DEmRNAs. (D-F) The horizontal axis of the heatmap indicates the samples, and the vertical axis of the heatmap indicates 15 significant DEGs. 
B
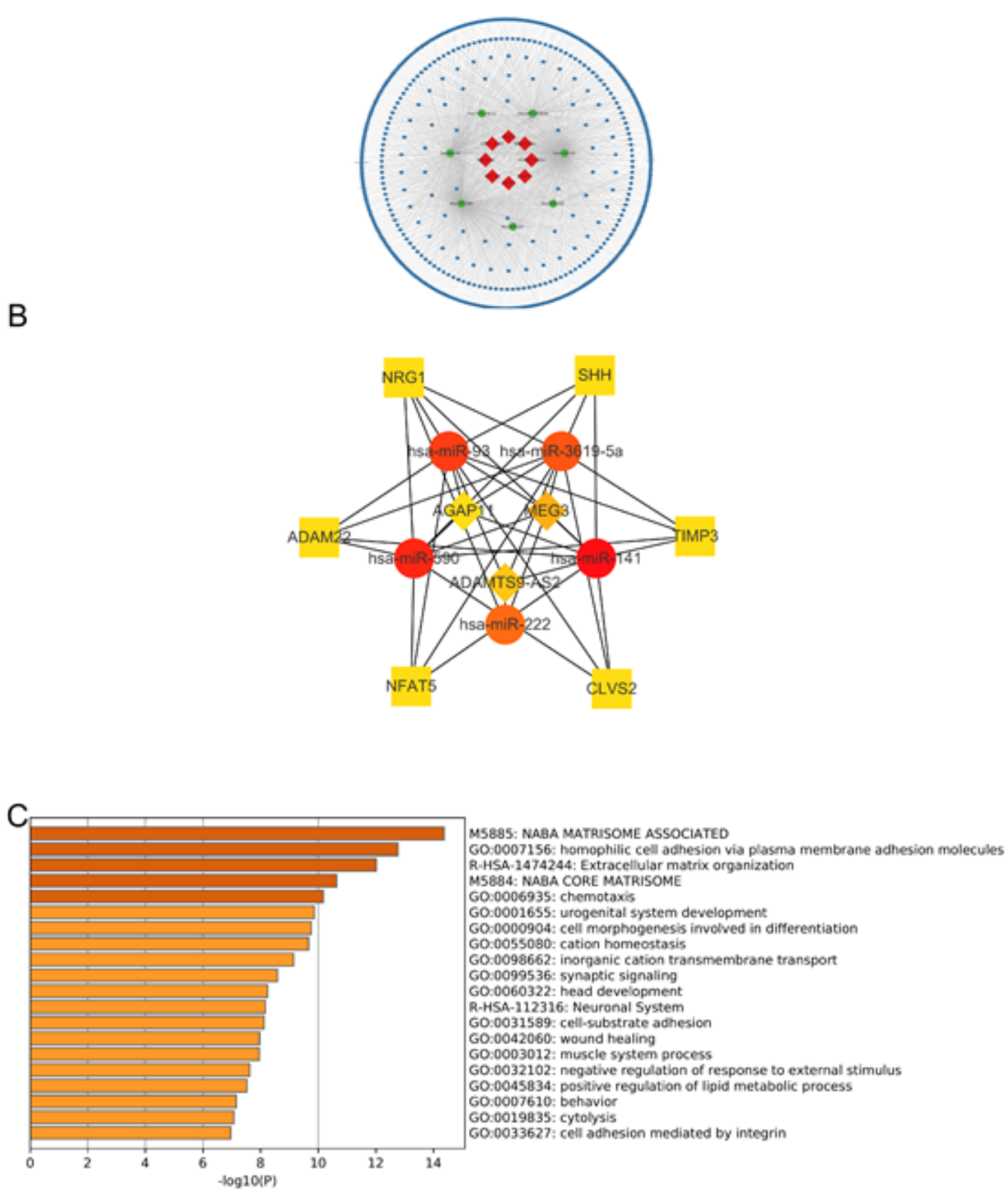

Figure 3

Construction and functional enrichment analysis of the InRNA-miRNA-mRNA triple regulatory network.

(A) The triple regulatory network in PRAD. The greens denote miRNAs, blues denote mRNAs, and reds denote IncRNAs. (B) Fourteen hub genes in this network with a score of $>2$. The ellipses denote miRNAs, rectangles denote mRNAs, and diamonds denote IncRNAs. (C) Functional enrichment analysis (GO and KEGG) of the DEmRNAs in the network. 
A
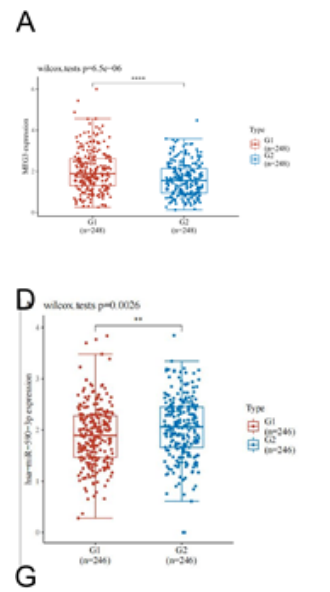

$B$
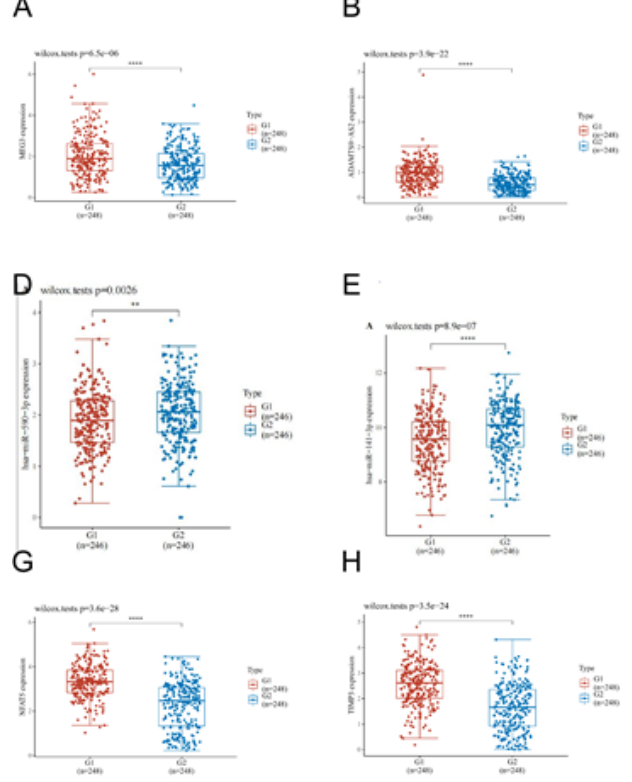

J

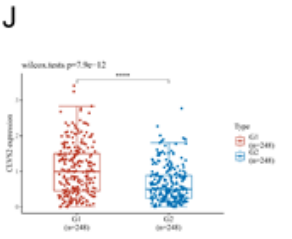

E

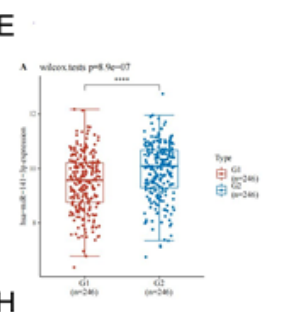

$\mathrm{H}$

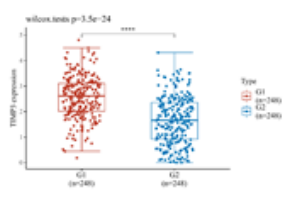

$\mathrm{K}$

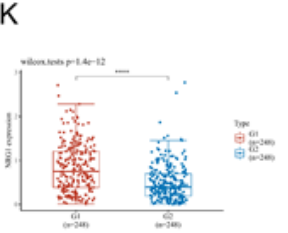

C
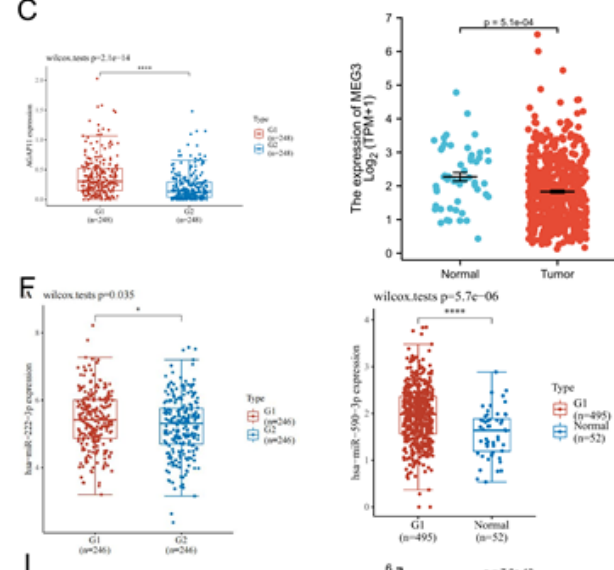

I

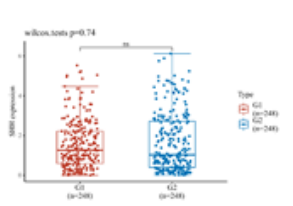

$\mathrm{L}$
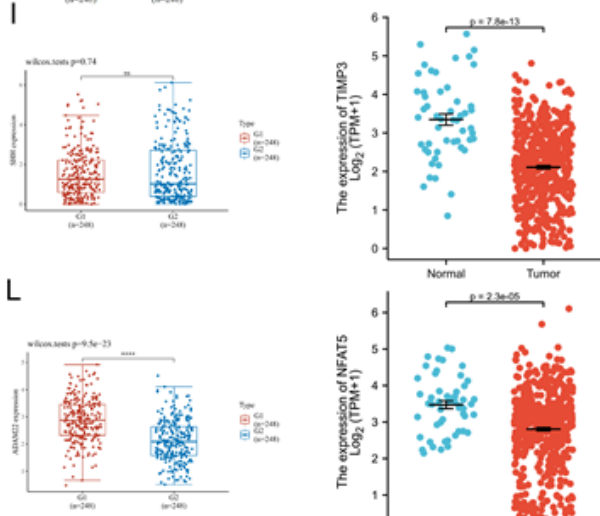
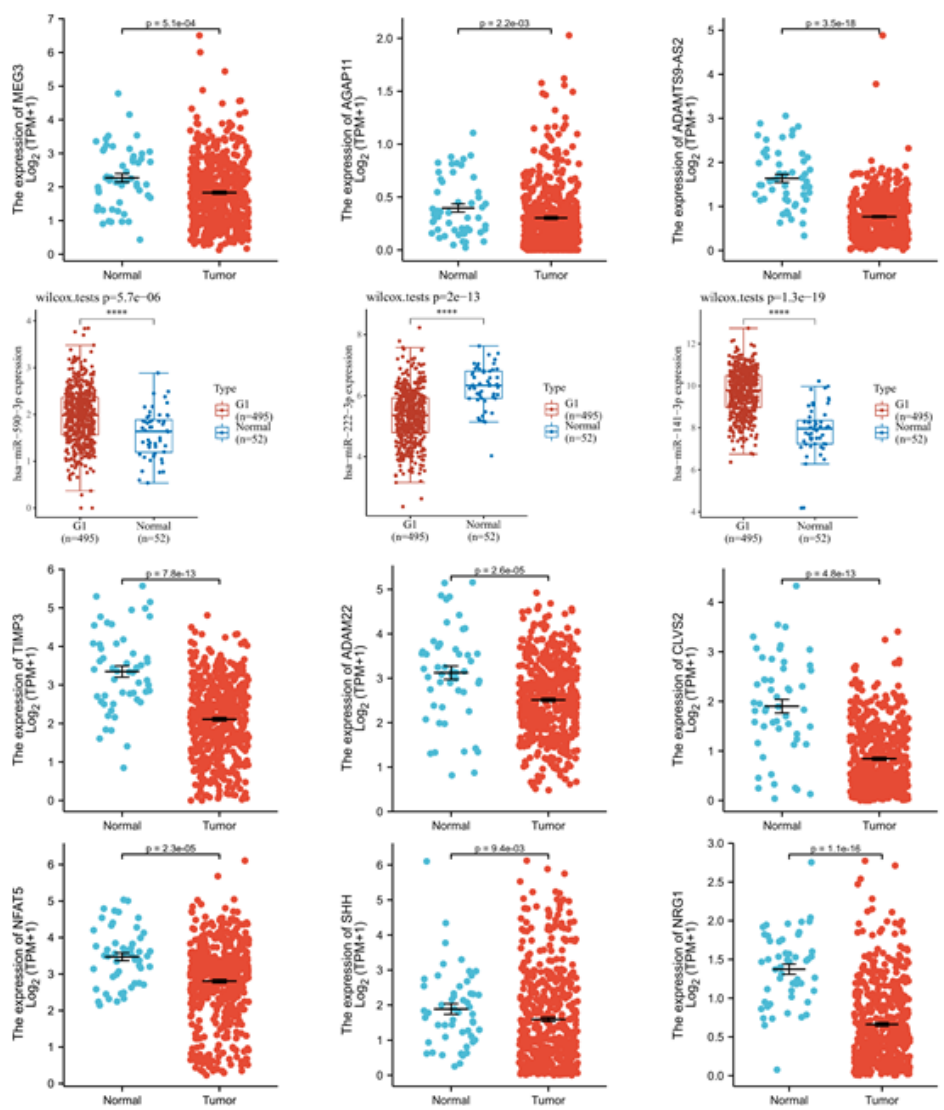

Figure 4

A. The distribution of 14 hub-RNA expression patterns from the triple regulatory network in TCGA PRAD dataset

(A-L) The expression patterns of three hub-DElncRNAs, five hub-DEmiRNAs, and six hub-DEmRNAs in PRAD samples with PTEN ${ }^{\text {high }}(\mathrm{G} 1)$ and PTEN ${ }^{\text {low }}(\mathrm{G} 2)$ expression groups.

B. The distribution of 14 hub-RNA expression patterns from the triple regulatory network in TCGA PRAD dataset

The expression patterns of three hub-DElncRNAs, five hub-DEmiRNAs, and six hub-DEmRNAs in PRAD and adjacent normal prostate tissues. $\mathrm{G} 1$ is the PRAD group and $\mathrm{G} 2$ is the adjacent normal prostate tissues. 

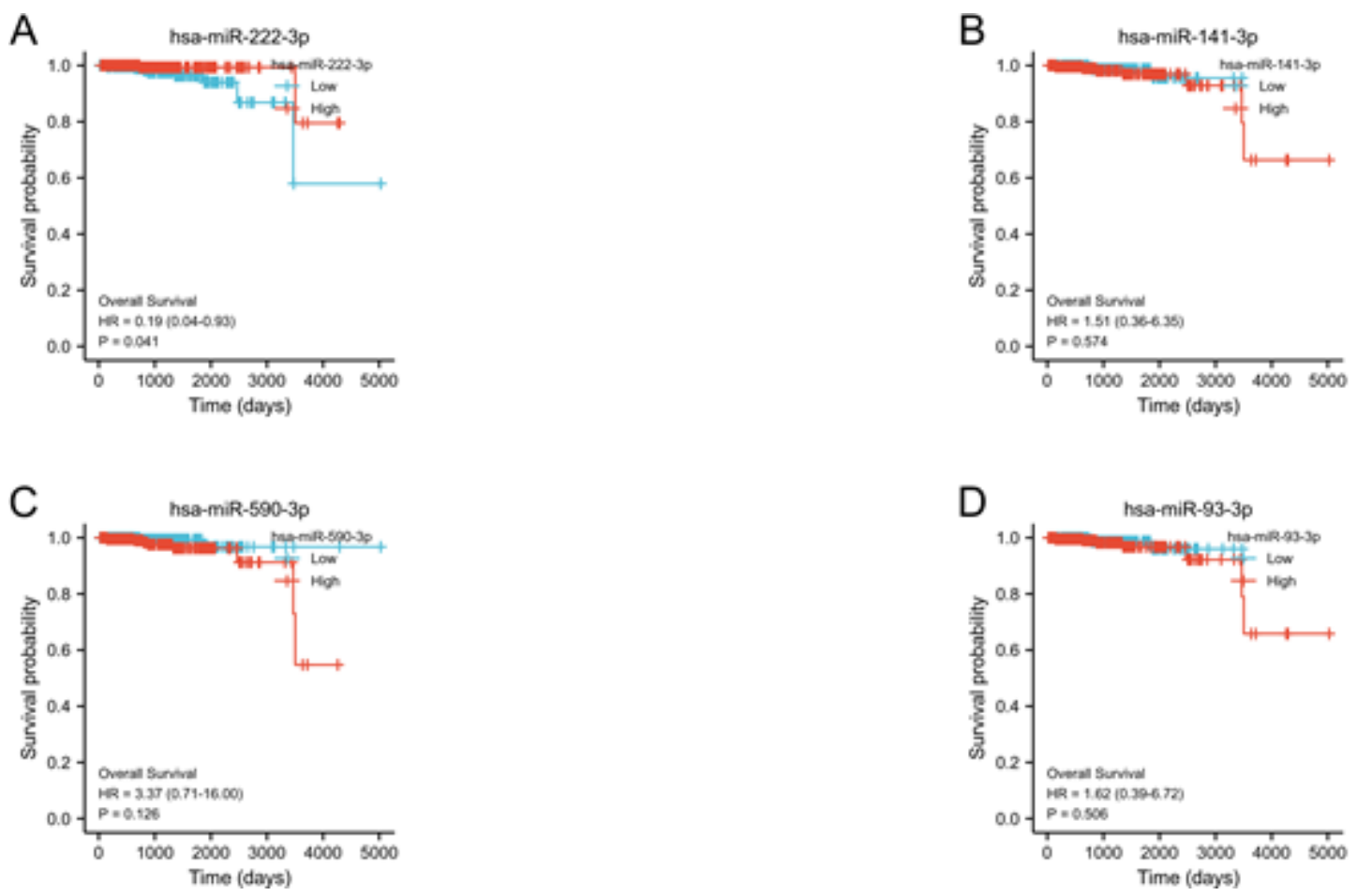

\section{Figure 5}

Overall survival analysis for the miRNAs in the hub triple regulatory network

(A-D) The high-and low-expression values of four hub-miRNAs were compared by a Kaplan-Meier survival curve for TCGA PRAD patient cohort. The horizontal axis indicates the overall survival time in days, and the vertical axis represents the survival rate. 
A

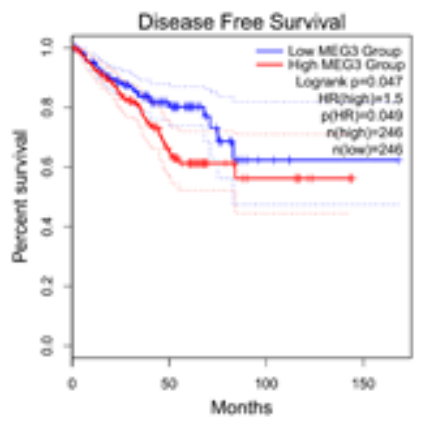

D

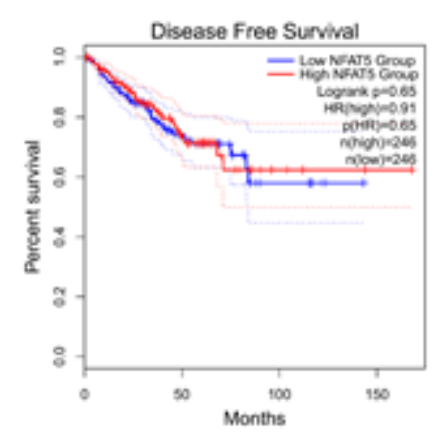

G

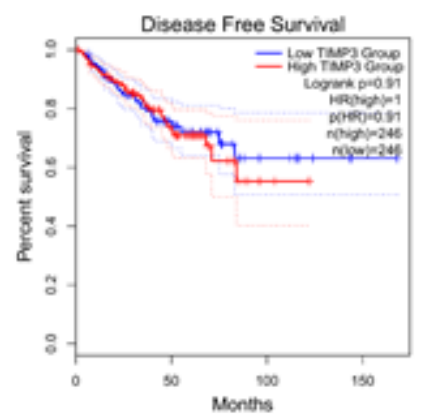

B

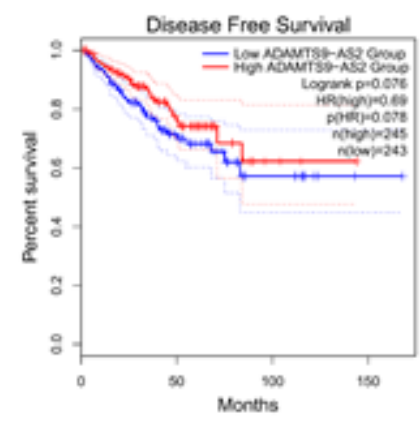

E

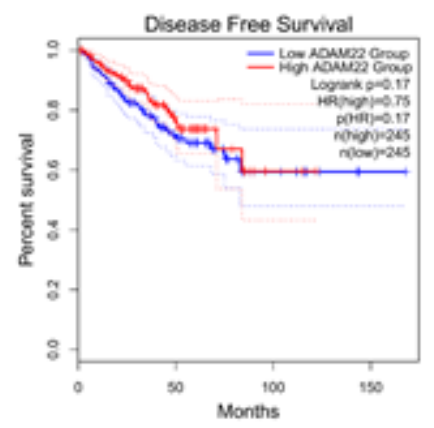

$\mathrm{H}$

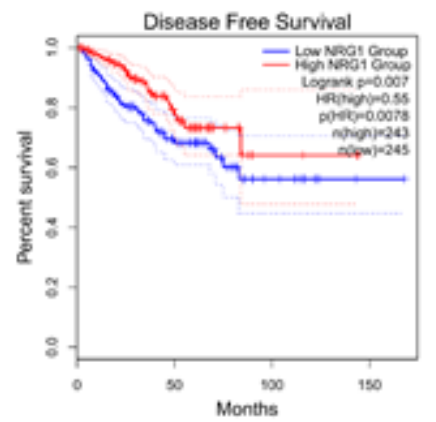

C

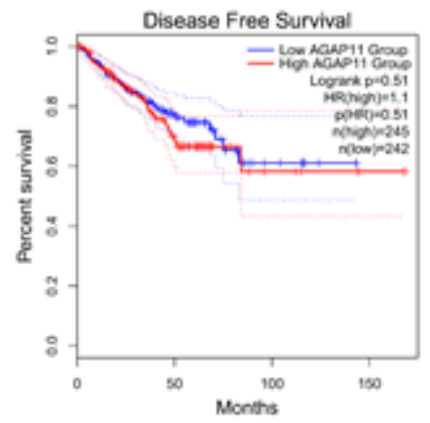

$\mathrm{F}$

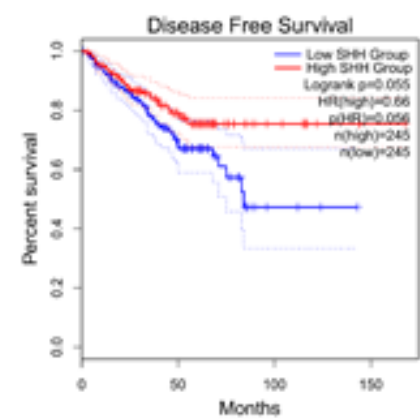

I

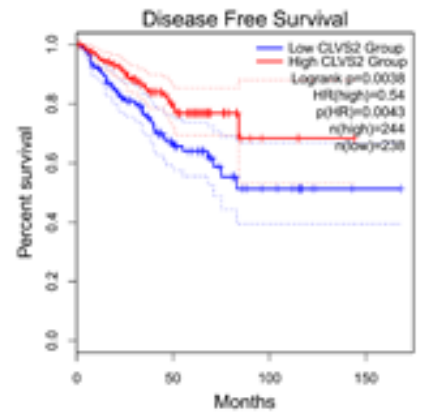

\section{Figure 6}

Disease Free survival analysis for the mRNAs and IncRNAs in the hub triple regulatory network

The high- and low-expression values of three hub-IncRNAs (A-C) and six hub-mRNAs (D-I) were compared by a Kaplan-Meier survival curve for TCGA PRAD patient cohort. The horizontal axis indicates the disease free survival time in months, and the vertical axis represents the survival rate. 
A
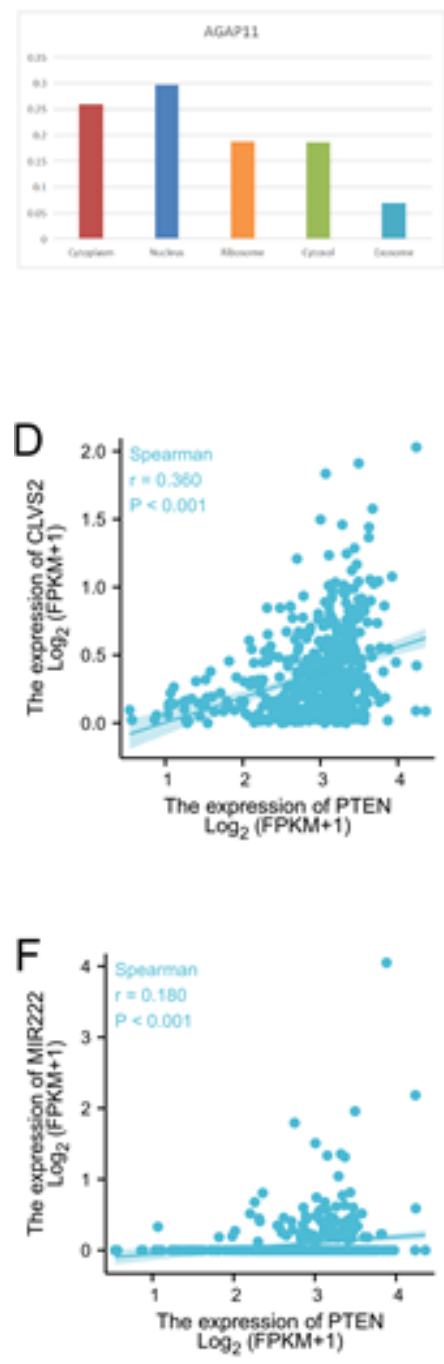

B

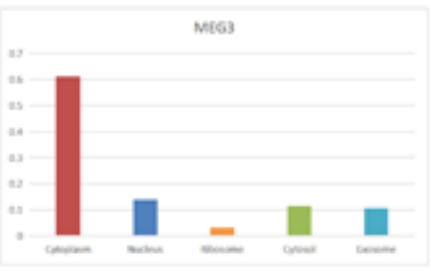

C

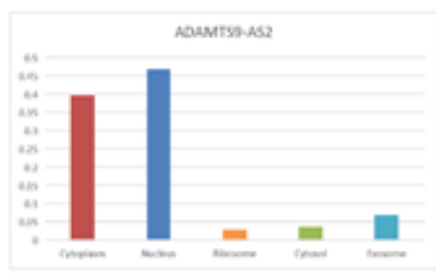

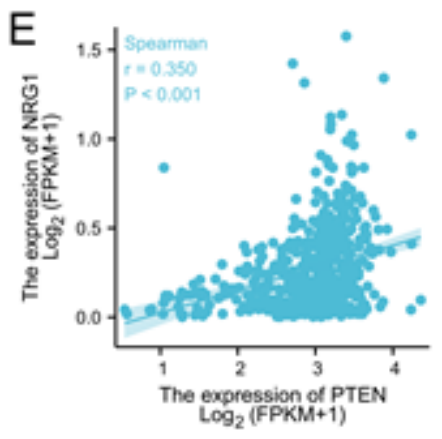

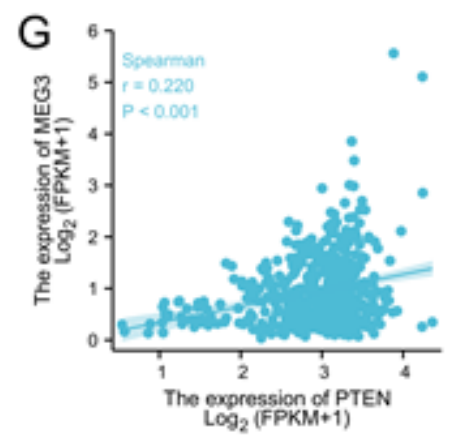

Figure 7

Construction and correlation analysis of the ceRNA network

(A-C) The cellular localization for three hub-IncRNAs (AGAP11, MEG3, and ADAMTS9-AS2) was predicted using IncLocator. (D) Correlation analysis between these four predictive RNAs and PTEN in PRAD. 

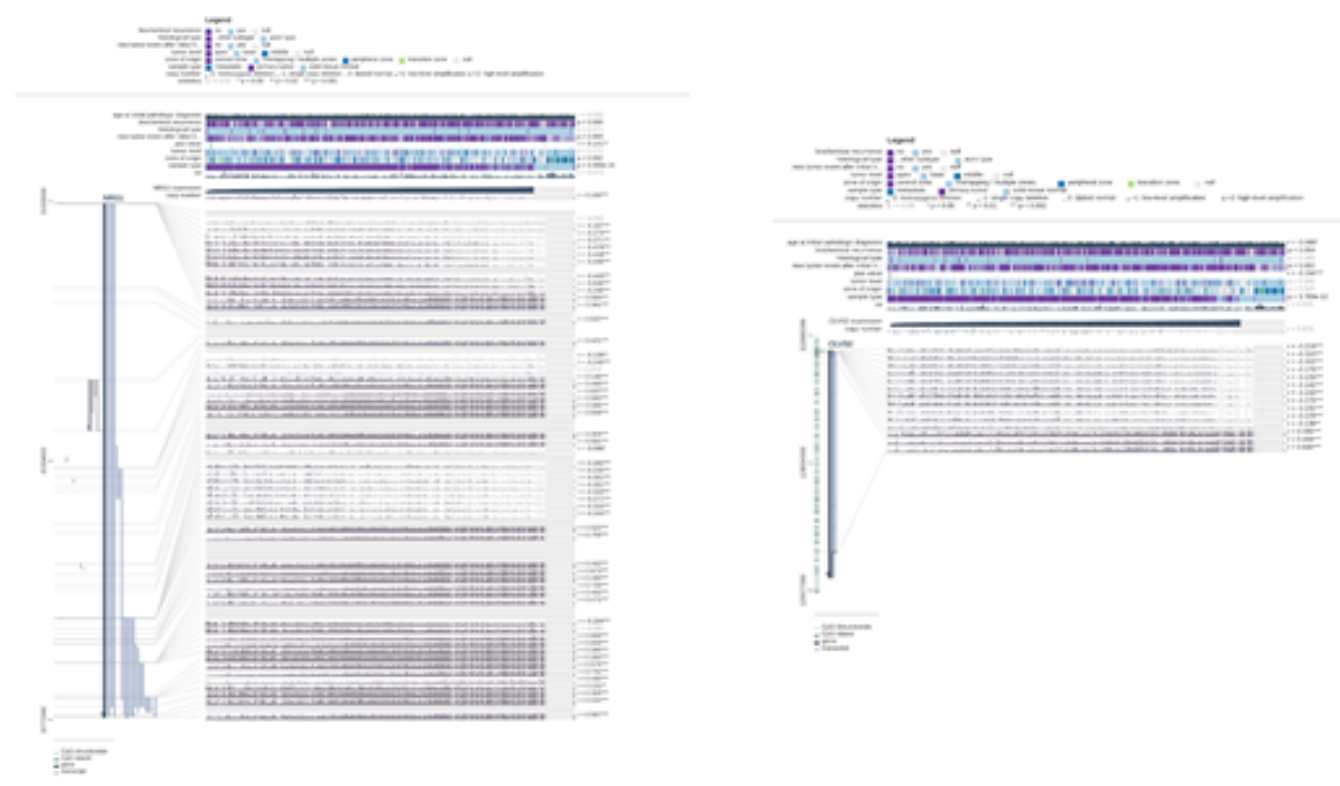

C

Promoter methylation level of NRC1 in PRAD

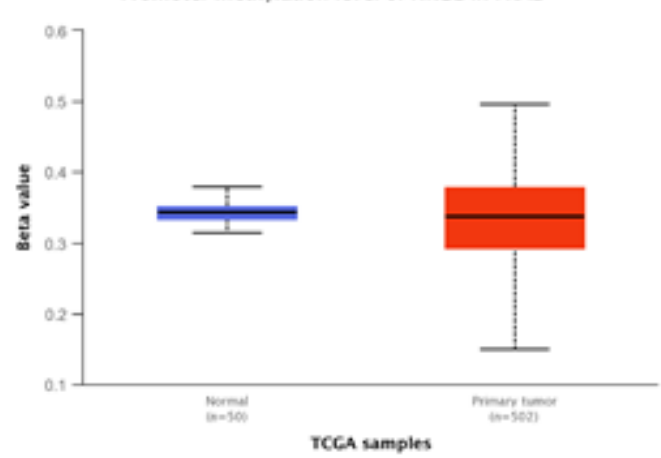

D

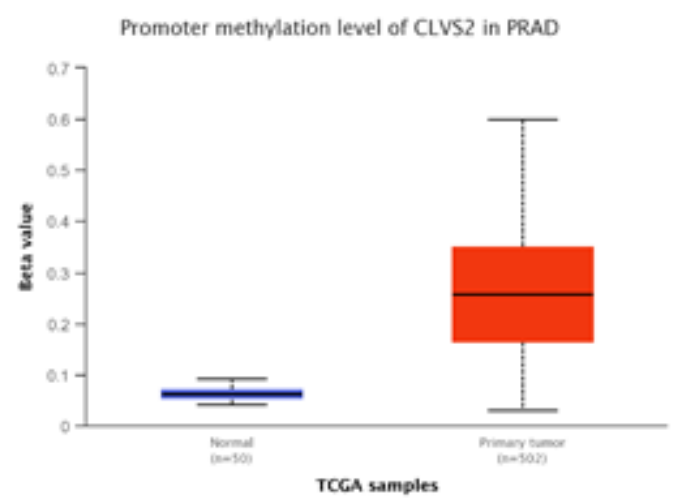

Figure 8

Methylation analysis of NRG1 and DDX

(A-B) The methylation site of NRG1(A) and CLVS2(B) DNA sequence association with gene expression was visualized using MEXPRESS. The expression of NRG1(A) and CLVS2(B) is illustrated by the blue line in the center of the plot. (C-D) Methylation of NRG1(C) and CLVS2(D) was evaluated using UALCAN. 
A
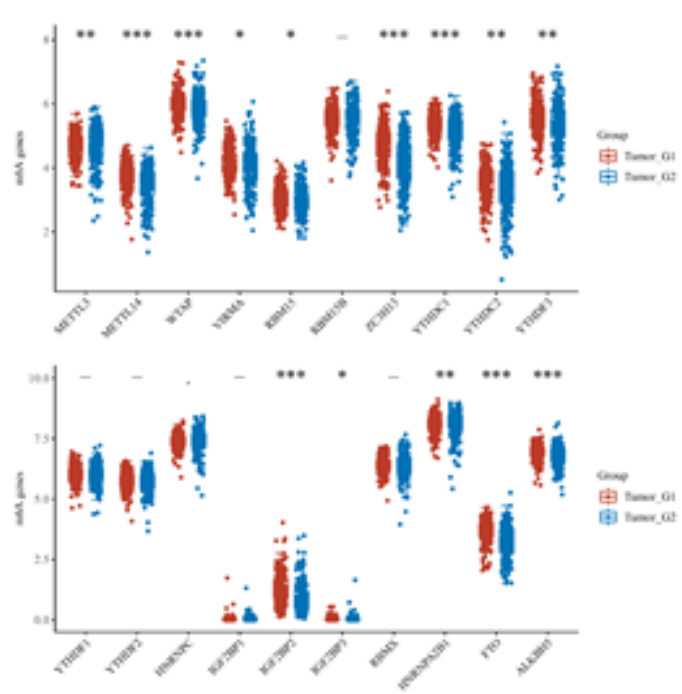

B
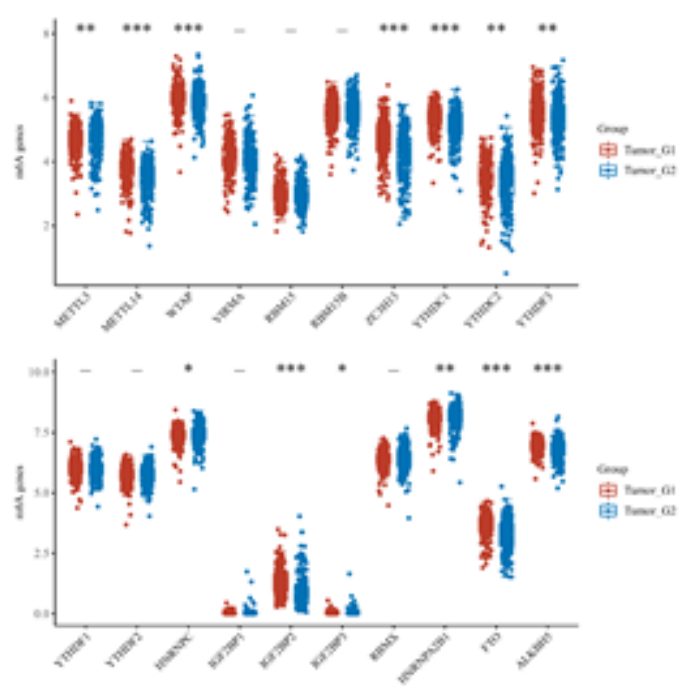

\section{Figure 9}

The difference of expression of m6A methylation genes in PRAD tissues with high and low NRG1(A) and CLVS2(B) gene expression. G1 is a high expression group and $\mathrm{G} 2$ is a low expression group. The horizontal axis represents different m6A methylation molecules, and the vertical axis represents the expression and distribution of the related gene, in which different colors represent different groups. * $\mathrm{P}<0.05, * \star \mathrm{P}<0.01, * \star * \mathrm{P}<0.001$. 
A

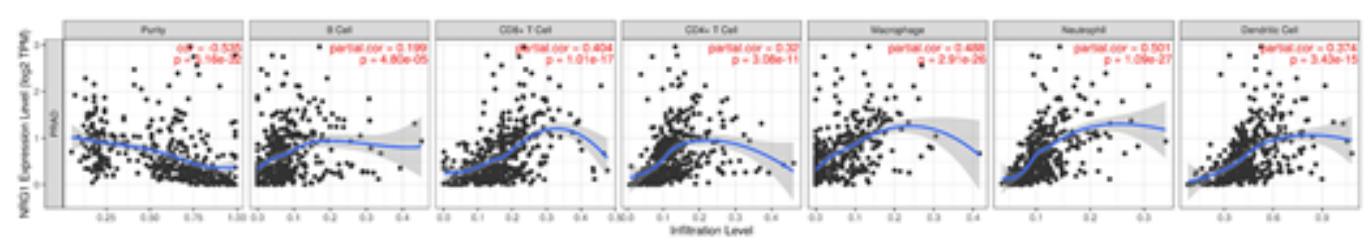

B

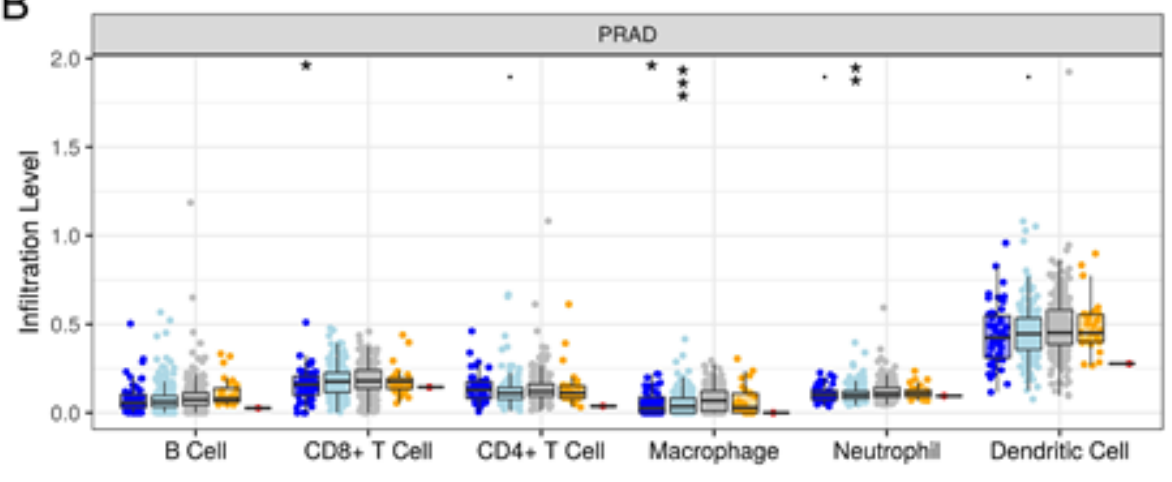

Copy Number

\& Deep Deletion

由 Arm-level Deletion

申 Diploid Normal

由 Arm-level Gain

申 High Amplication

C

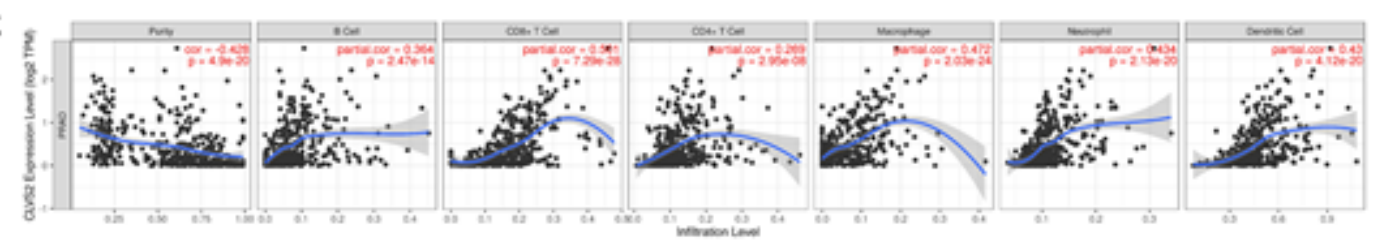

D

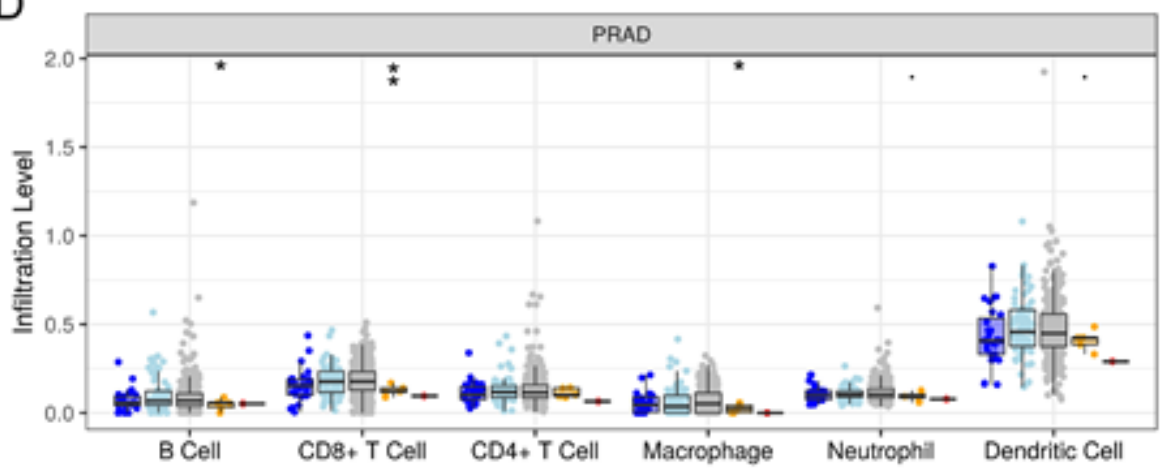

Copy Number

由 Deep Deletion

由 Arm-level Deletion

由 Diploid Normal

申 Arm-level Gain

申i High Amplication

Figure 10

Correlation analysis of NRG1 and CLVS2 expression and immune infiltration in PRAD

Association between NRG1(B) and CLVS2(D) gene copy number and immune cell infiltration levels in PRAD cohorts. (A and C) Correlation of NRG1(A) and CLVS2(C) expression with immune infiltration level in PRAD. 

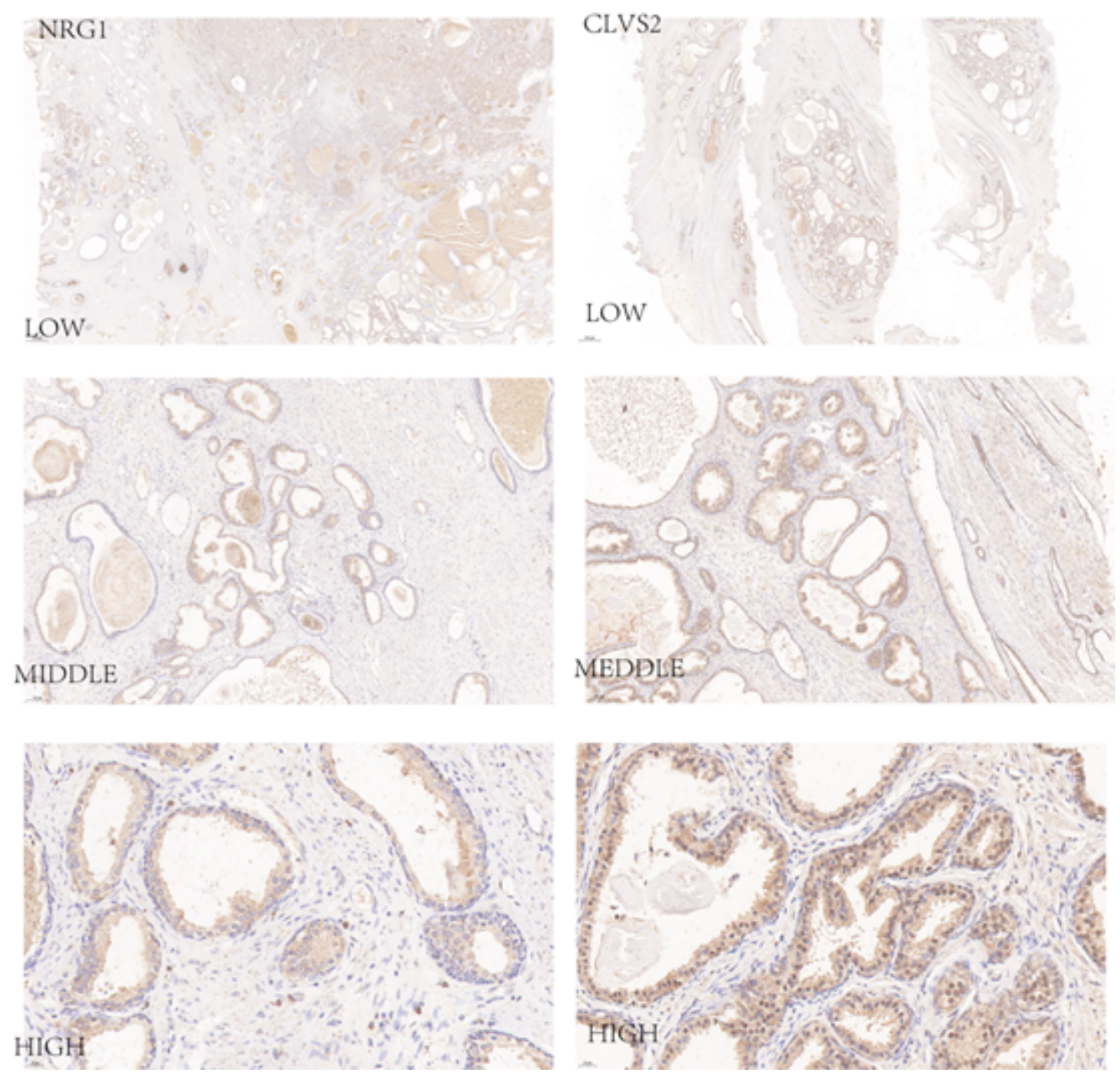

Figure 11

Immunohistochemical study of NRG1 protein and CLVS2 protein in patients with prostate cancer 